\title{
Impact of evolving practices on SARS-CoV-2 positive mothers and their newborns in the largest public healthcare system in America
}

\author{
Yogangi Malhotra $\mathbb{1}^{1} \cdot$ Clementine Knight $\mathbb{1}^{2} \cdot$ Uday P. Patil $\mathbb{1}^{3} \cdot$ Harry Sutton ${ }^{1} \cdot$ Taneisha Sinclair $\mathbb{D}^{2}$. \\ Max C. Rossberg ${ }^{2}$ - Arpit Gupta $\mathbb{D}^{4} \cdot$ Kathryn Whitehead ${ }^{5} \cdot$ Tianying $\mathrm{Li}^{5} \cdot$ Daryl Wieland $\mathbb{D}^{1} \cdot$ Ivan Hand $\mathbb{D}^{6}$
}

Received: 4 July 2020 / Revised: 2 February 2021 / Accepted: 17 February 2021 / Published online: 5 March 2021

(c) The Author(s), under exclusive licence to Springer Nature America, Inc. 2021

\begin{abstract}
Objective The impact of evolving guidelines and clinical practices on SARS-CoV-2-positive dyads across New York City Health and Hospitals during the early peak of COVID-19.

Design A retrospective cohort study of positive-positive (P/P), positive-negative (P/N), and positive-untested (P/U) dyads delivered from March 1 to May 9, 2020. Wilcoxon rank sum, Chi-squared, and Fisher exact tests were used to analyze demographics, clinical variables, and system-wide management practices.

Result A total of 2598 mothers delivered. 23.8\% (286/1198) of mothers tested for SARS-CoV-2 were positive. 89.7\% (260/ 290) newborns of SARS-CoV-2-positive mothers were tested and 11 were positive. Positive-positive newborns were more likely to be breastfed (81\%), be admitted to NICU, and have longer length of stay ( 7.5 days) than P/N and P/U newborns. Conclusion Our study shows that varied testing, feeding, and isolation practices resulted in favorable short-term outcomes for SARS-CoV-2-positive mothers and their newborns. High-risk populations can be safely and effectively treated in resource-limited environments.
\end{abstract}

\section{Introduction}

In March 2020, New York City began to see a surge in cases of SARS-CoV-2, including pregnant women [1]. New York City Health + Hospitals $(\mathrm{NYC} \mathrm{H}+\mathrm{H})$, the largest municipal healthcare system in the country delivering $\sim 16,000$ pregnancies in 11 acute care facilities annually,

These authors contributed equally: Yogangi Malhotra, Clementine Knight

Yogangi Malhotra

malhotry@nychhc.org

NYC Health + Hospitals/Jacobi, Bronx, NY, United States

2 Albert Einstein College of Medicine, Bronx, NY, United States

3 NYC Health + Hospitals/Elmhurst, Queens, NY, United States

4 NYC Health + Hospitals/Metropolitan, New York, NY, United States

5 NYC Health + Hospitals/Office of Quality and Safety, New York, NY, United States

6 NYC Health + Hospitals/King's County, Brooklyn, NY, United States became the epicenter during this surge. Uncertainty existed regarding the impact of the COVID-19 infection on management of pregnant women and newborns.

Emerging literature and anecdotal experience suggested that a significant number of SARS-CoV-2 positive women were asymptomatic on their admission for delivery [1-3]. As information about the disease became available, guidelines emerged to identify and manage pregnant women during admission for delivery, safely cohort positive patients, appropriately isolate newborns, protect staff, and preserve personal protective equipment. Our 11 facilities adapted to the evolving literature and national guidelines around measures such as universal testing, mode of delivery, isolation recommendations, exposure of newborns to SARS-CoV-2 positive mothers, and discharge planning [4-8]. Despite the emerging data on SARS-CoV-2, reports on newborns who are positive for the virus remain limited, and the effects of infection on newborns is still poorly understood $[9,10]$. On the other hand, strong historical evidence supports early skin-to-skin contact after delivery, breastfeeding, and rooming in to initiate early bonding between mothers and newborns. Furthermore, studies evaluating other viral pathogens such as SARS and MERS showed no increased negative outcomes in maintaining these practices in the setting of infection [11, 12]. 
Fig. 1 Mother/newborn dyads. Flowchart of delivered and tested mother/newborn dyads in $\mathrm{NYCH}+\mathrm{H}$ facilities between March 1 and May 9, 2020.

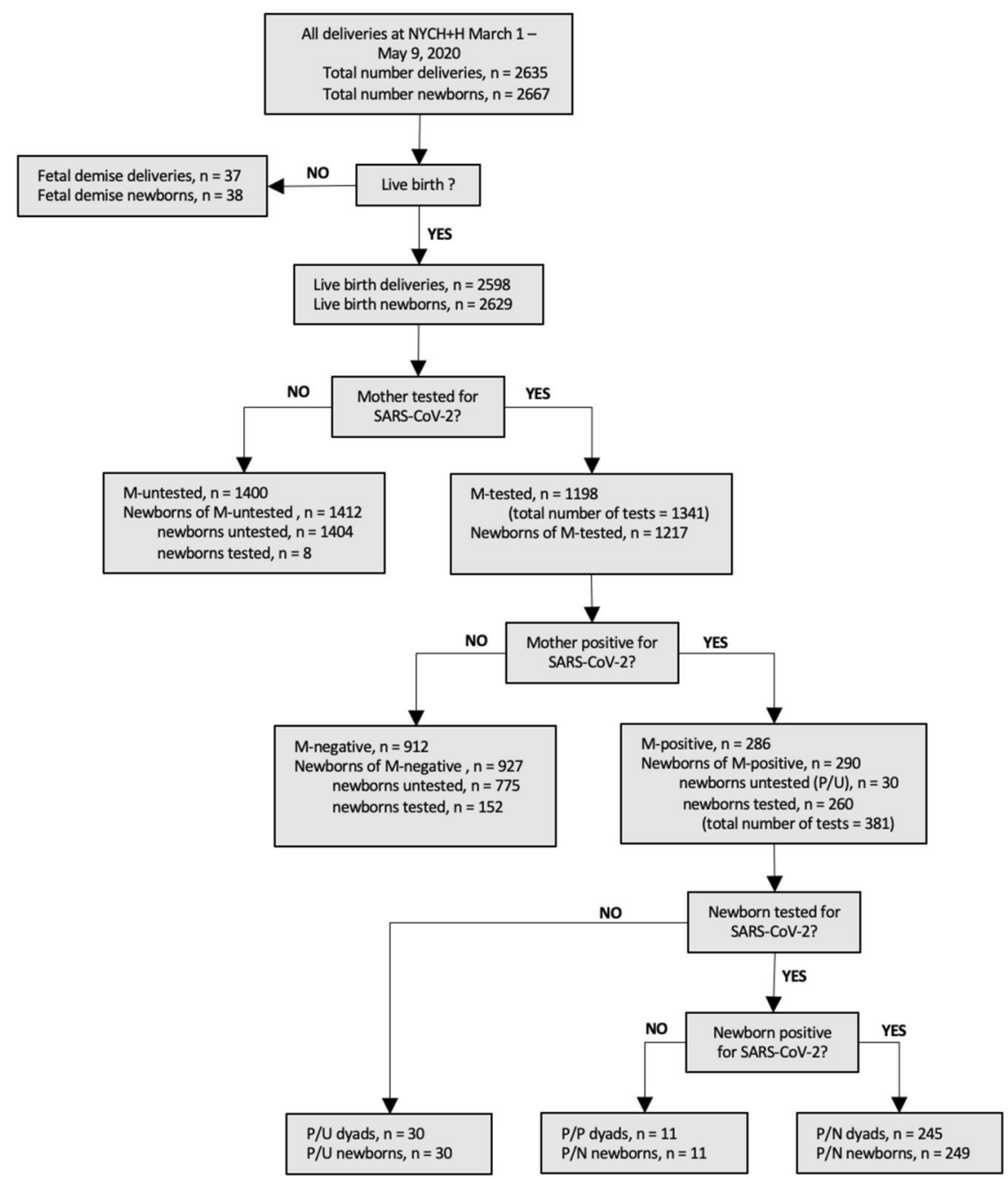

Our objective was to study the impact of the evolving approaches on the management of mother/baby dyads that were affected by maternal SARS-CoV-2 status across our 11-hospital healthcare system. Our study compares the characteristics and outcomes of the SARS-CoV-2 positive mothers (M-positives) and the three cohorts defined by newborn test results, namely, the positives, the negatives, and the untested. We specifically looked at metrics that were most impacted by maternal test status: mode of delivery, length of stay (LOS), NICU admission, skin-toskin, breastfeeding, and newborn infection rates.

\section{Methods}

\section{Study design, location, and approval}

We identified all mother-newborn dyads delivered from March 1 to May 9, 2020 at any of the $11 \mathrm{NYCH}+\mathrm{H}$ hospitals that had maternal SARS-CoV-2 testing done prior to or during the delivery encounter. Inclusion criteria were all pregnant women who had live birth deliveries (singleton and multiples) within the $\mathrm{NYC} \mathrm{H}+\mathrm{H}$ system during the study period (Fig. 1). Average baseline data for breastfeeding and skin-to-skin was collected for the period of Mar 1, 2019 - Feb 29, 2020.

The tested mothers fell into two groups, namely the Maternal SARS-CoV-2 test positive (M-positive) and Maternal SARS-CoV-2 test negative (M-negative). The mothers who were not tested during this time were denoted M-untested.

A mother was designated "positive" if any test ordered and collected prior to or during the delivery encounter had a positive result. The same criteria were used for newborn designation with their testing period anywhere from birth to discharge from the hospital.

Exclusion criteria were dyads with mothers only tested outside the NYC $\mathrm{H}+\mathrm{H}$ system and those tested within the NYC $\mathrm{H}+\mathrm{H}$ system but delivered at outside hospitals. Patients with missing data were excluded from 
specific analyses for those specific parameters. Testing of mothers and newborns collected after discharge from the delivery encounter was also excluded. Data were obtained through retrospective review of the system-wide electronic medical records (EMR). This study was approved by the $\mathrm{NYC} \mathrm{H}+\mathrm{H}$ institutional review board.

\section{Testing specifications}

Four rt-PCR tests were employed during this period. The majority of both mother and newborn testing was done using either the BioReference real-time RT-PCR assay (BioReference, the Bioreference Laboratories Inc, Spring Valley, NY, USA) or the Cepheid Xpert Xpress SARSCoV-2 test (Cepheid, Sunnyvale, CA, USA) [13, 14]. The DOHMH and LabCorp tests were used in small numbers primarily during the first few weeks and were provided by the New York State Department of Health and Mental Hygiene (DOHMH) [15]. Testing was performed on oroor nasopharyngeal swab specimens.

\section{Group stratification}

For this study we analyzed all M-positive dyads (positivemother/newborn) and further stratified them based on newborn test results as: positive/positive (P/P), positive/ negative $(\mathrm{P} / \mathrm{N})$, and positive/untested $(\mathrm{P} / \mathrm{U})$.

\section{Outcomes and statistical analyses}

We collected maternal demographics on age, race/ethnicity, BMI, comorbidities (asthma, hypertension, and diabetes), type of health insurance, and home location by NYC borough. Maternal clinical data was collected on gravidity, fever, chest imaging, respiratory support, duration of rupture of membranes, route of delivery, breastfeeding, skin-toskin, and length of hospital stay for their delivery encounter. Newborn clinical data was collected on gestational age, birth weight, Apgar score, respiratory support, test result for SARS-CoV-2, location, and length of stay. The outcomes of interest were route of delivery, Apgar scores, skin-to-skin, breastfeeding, admission to NICU, isolation, and length of stay for the dyads.

We compared demographic and clinical variables among the stratified M-positive sub-categories: $\mathrm{P} / \mathrm{P}, \mathrm{P} / \mathrm{N}$, and $\mathrm{P} / \mathrm{U}$. Continuous variables (ages, time duration) were analyzed using the independent sample $t$ test or Kruskal Wallis test depending on the distribution of the data. Categorical variables were analyzed using Fisher's exact test due to the small sample size for the P/P dyad. Analyses were done using SAS 7.1. All tests were two-sided. $P<0.05$ were considered statistically significant.

\section{Results}

From March 1 to May 9 2020, NYCH + H delivered 2629 live-born newborns to 2598 women. Of those women, $46 \%$ (1198/2598) were tested for SARS-CoV-2 during or just prior to the delivery encounter. $286 \mathrm{M}$-positives gave birth to 290 live newborns.

\section{SARS-CoV-2 testing and positivity}

During this period 1341 SARS-CoV-2 rt-PCR tests were performed on 1198 delivering mothers.

A total of $93 \%$ of the mothers were tested once during their hospital course, while the remaining underwent 2-6 tests. $40 \%(534 / 1341)$ of these tests were Bioreference and 59\% (789/1341) were Cepheid. The turnaround time for maternal Bioreference tests was $63 \mathrm{~h}$ and for Cepheid was $4 \mathrm{~h}$.

$24 \%(286 / 1198)$ of all tested patients were positive (combined pre- and post-universal testing periods). The prevalence of SARS-CoV-2 among pregnant women in our population during the universal testing period (Apr 12-May 9) was $19 \%$, dropping from $27.1 \%$ at the peak of the surge to $13.9 \%$ by May 9 (Fig. 2).

90\% (260/290) of the M-positive newborns were tested for SARS-CoV-2 prior to discharge from hospital and $4.2 \%(11 / 260)$ were positive. Of these 11 positive newborns, 10 had 1 positive test and 1 newborn had 7 positive tests.

$58.8 \%(153 / 260)$ of M-positive newborns were tested only once before discharge and $41.2 \%$ were tested two or more times. No other tested infants (those born to Mnegative or M-untested) had a positive test result before discharge. 10\% (30/290) of the newborns of M-positives were discharged without being tested $(\mathrm{P} / \mathrm{U})$ due to either prolonged maternal testing turnaround time or shortage of supplies.

381 tests were performed on 260 newborns of Mpositives. $74 \%$ (281/381) of these were Bioreference and $26 \%$ were Cepheid. The turnaround time for newborn Bioreference was $68 \mathrm{~h}$ and for Cepheid was $3 \mathrm{~h}$. The overall average time to the first SARS-CoV-2 test for newborns was $20 \mathrm{~h}$ (range $0.4-130 \mathrm{~h}$ ). For P/P dyad newborns the average was $13 \mathrm{~h}$ vs. $20 \mathrm{~h}$ for $\mathrm{P} / \mathrm{N}$ newborns $(p=0.1327)$.

\section{Demographics and characteristics}

When comparing our two most populous patient groups, the Latino/Hispanic group had a significantly higher positivity rate than the Black/Non-Hispanics (33\% vs. 19\%; $p=$ 0.0003). Most M-positives resided in Queens (39\%), 
Fig. 2 SARS-CoV-2 rt-PCR testing. Testing of (A) Mothers and (B) Newborns at NYC H+ $\mathrm{H}$ facilities by week between March 1 and May 9, 2020. Total number of individuals tested and number who tested positive. Total number of tests performed by type.

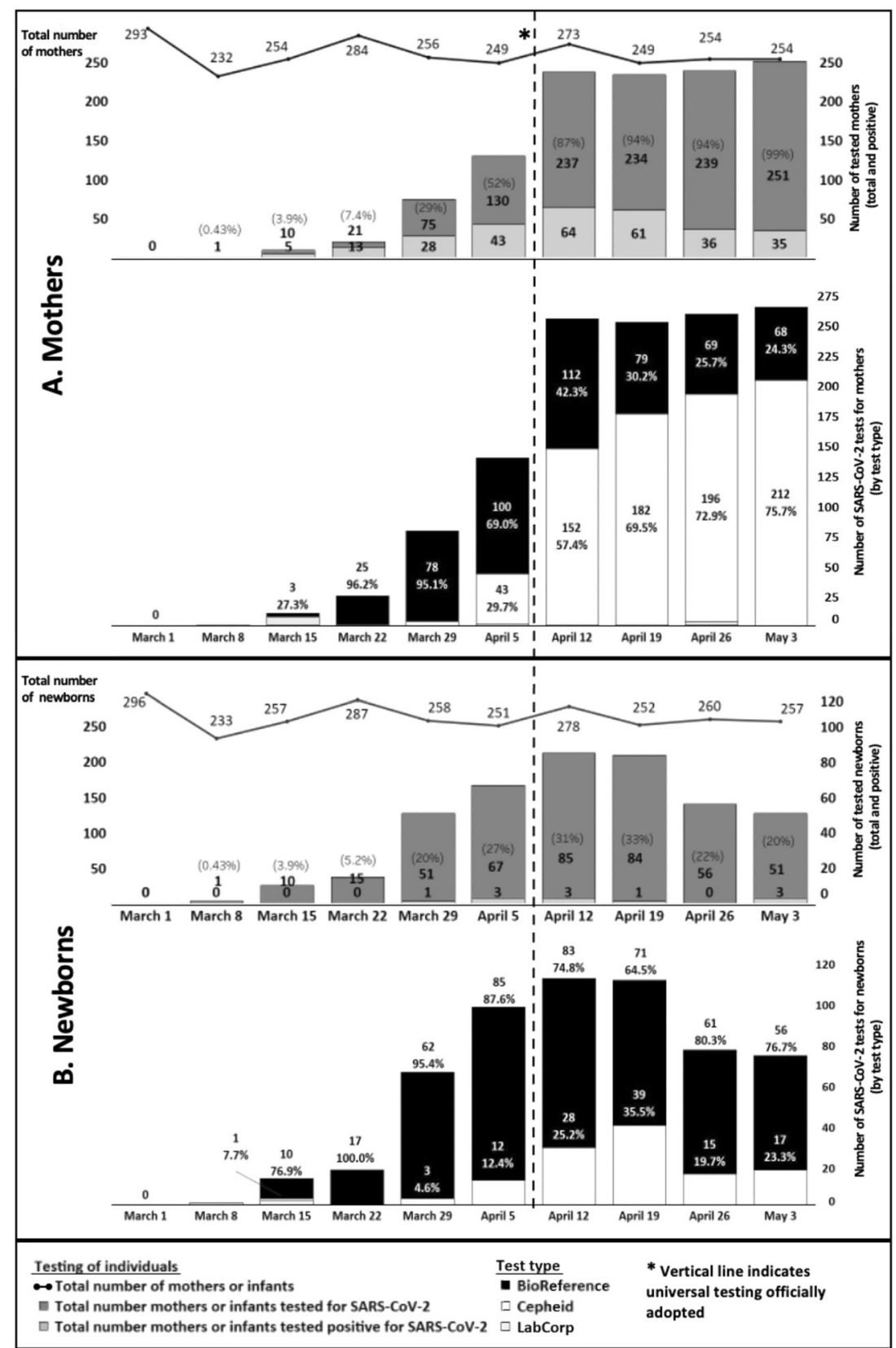

followed by the Bronx (29\%), Brooklyn (29\%), and then Manhattan $(3 \%)$. The positivity rate did not significantly differ by the insurance carrier.

Multigravida women were more likely to test positive than primigravida women $(27 \%$ vs. $18 \% ; p=0.0022) .93 \%$ of our patients were obese $(59 \%)$ or overweight $(34 \%)$. In our population $31 \%$ of the patients had only one of the three comorbidities analyzed, namely asthma, hypertension, or diabetes. Patients with an asthma diagnosis had a significantly lower positivity rate than the non-asthmatics (15\% vs. $25 \% ; p=0.0127)$.

Nine M-positives were admitted to the ICU, but none of their newborns tested positive for SARS-CoV-2.

\section{Cohort Comparison of $P / P, P / N$, and $P / U$ dyads}

The median age of all M-positives was 29 years, with mothers of $\mathrm{P} / \mathrm{P}$ dyad slightly older at 33 years $(p=0.1967)$. There was no statistically significant difference in BMI, comorbidities, or insurance carriers between the three Mpositive dyad sub-groups. Fisher exact analysis could not be performed for the borough distribution and race/ethnicity because of the small $\mathrm{n}$ for the P/P cohort.

The mothers in the $\mathrm{P} / \mathrm{N}$ cohort were more likely to have fever $(p=0.07)$ and more likely to undergo chest imaging $(p=0.03)$. The need for respiratory support was not significantly different between these cohorts $(p=0.73)$. The 
mothers in the $\mathrm{P} / \mathrm{P}$ cohort did not have a significantly higher cesarean delivery rate $(p=0.63)$ than $\mathrm{P} / \mathrm{N}$ and $\mathrm{P} / \mathrm{U}$. The mothers in the $\mathrm{P} / \mathrm{U}$ cohort had the shortest median length of stay $(p=0.19)$.

$\mathrm{P} / \mathrm{P}$ newborns were more likely to be admitted to the NICU for isolation or medical indication. The overall length of stay for $\mathrm{P} / \mathrm{P}$ newborns (7.5 days) was significantly longer than $\mathrm{P} / \mathrm{N}$ (5 days) and $\mathrm{P} / \mathrm{U}$ newborns (2.5 days; $p=0.004$ ). The average NICU length of stay and need for respiratory support of all three cohorts was similar. There were no other statistically significant differences between these cohorts across all the other discussed metrics (Table 1).

In our patients during this period, the overall rate of breastfeeding among M-positives was 57\% dropping from $\mathrm{NYCH}+\mathrm{H}$ 's system-wide baseline (March 1, 2019 February 29, 2020) of $87 \%$. Similarly, the M-positive skin-to-skin rate of $36 \%$ was lower than the baseline of $60 \%$. The skin-to-skin rate was comparable for the $\mathrm{P} / \mathrm{P}, \mathrm{P} /$ $\mathrm{N}$, and $\mathrm{P} / \mathrm{U}$ dyads. The breastfeeding rate for $\mathrm{P} / \mathrm{P}$ dyads was higher (81\%) than $\mathrm{P} / \mathrm{N}(55 \%)$ and $\mathrm{P} / \mathrm{U}(70 \%)$ dyads but did not reach statistical significance due to the small number of $\mathrm{P} / \mathrm{P}$ dyads. When compared between the $\mathrm{P} / \mathrm{P}, \mathrm{P} /$ $\mathrm{N}$, and $\mathrm{P} / \mathrm{U}$ dyads, the skin-to-skin rates were similar. The breastfeeding rate for $\mathrm{P} / \mathrm{P}$ dyads was higher $(81 \%)$ than $\mathrm{P} / \mathrm{N}(55 \%)$ and $\mathrm{P} / \mathrm{U}(70 \%)$ dyads but did not reach statistical significance due to the small number of $\mathrm{P} / \mathrm{P}$ dyads. The weekly variation in these practices is illustrated in Fig. 3.

\section{Additional characteristics of the P/P dyads}

The timing and results of SARS-CoV-2 testing for P/P dyads are illustrated in Fig. 4.

All P/P dyad mothers had normal leukocyte count $(4300-15,000 / \mathrm{mcL})$ [16]. Seven mothers were asymptomatic. Four had significant medical histories or presented with mild respiratory symptoms and elevated inflammatory markers [17]. One presented with disseminated intravascular coagulation. None were admitted to the ICU.

10 out of 11 newborns were delivered at term, were appropriate for gestational age and needed no resuscitation. Two presented with mild respiratory distress after day of life (DOL) 1 that resolved without intervention. The length of stay ranged from 2 to 5 days with prolonged stay for pending rt-PCR results or foster care placement, and not due to COVID-19 related symptoms.

Two newborns were admitted to the NICU for medical indications other than isolation. One of these two babies was believed to have severe symptoms related to COVID-19 infection and had a prolonged hospital course [18].

\section{Discussion}

NYC $\mathrm{H}+\mathrm{H}$ cares for a diverse population. Over $30 \%$ of our patients are uninsured, and over $60 \%$ self-identify as either Black/Non-Hispanic, Latino/Hispanic, or Asian/NonHispanic. These women are among the highest risk groups for pregnancy related morbidity as well as SARS-CoV-2 infection [19].

As cases of COVID-19 surged in New York, our healthcare system was at the epicenter of the pandemic from March through May 2020. The guidelines around the management of pregnant women admitted for delivery and their newborns were evolving rapidly [4].

This study describes the impact of these evolving approaches for mother-baby dyads affected by maternal COVID-19 across the 11 hospitals of our healthcare system.

Five metrics would have likely been affected by maternal positive and/or pending test results: breastfeeding, skin-toskin, maternal LOS, newborn NICU admission, and newborn LOS. These metrics varied among $\mathrm{NYCH}+\mathrm{H}$ hospitals based on hospital resources and protocols and were affected by availability of maternal testing, timing of testing relative to delivery, and the turnaround time to obtain the results (Fig. 3). Balancing the potential risks of exposure or transmission between infected mothers and their newborns with proven benefits from initiation of bonding and breastfeeding was a clinical challenge facing the perinatal community during the pandemic.

The early rapid surge in Brooklyn and Queens resulted in a real crisis with limited guidance. It precipitated the early adoption for universal testing in these two boroughs. By the third week of April, over $90 \%$ of our patients were being tested prior to delivering their newborn [8]. The vast number of tests leading up to this period were Bioreference. Each facility adapted to the long turnaround time. One solution early in the surge, was to adopt a policy of bathing and default testing newborns of persons under investigation (PUIs) in the first few hours before reuniting them with their mothers. This practice allowed potential horizontal transmission to be ruled out as the source of infection if both tests came back positive without further delaying mother-newborn interactions such as rooming in and breastfeeding [20]. Other facilities, with less testing access and a later surge, adopted isolation protocols for newborns of PUIs. These measures were implemented based on available space and mechanical resources with solutions ranging from use of incubators for co-located newborns, to use of isolation rooms with HEPA filters, to non-clinicallyindicated NICU admission for the newborn. The Cepheid test became more widely available in mid-April (Fig. 2). Its significantly shorter turnaround time helped solve some of the problems associated with BioReference, hence it was used whenever possible. To this end, mothers were 
Table 1 Demographic characteristics and clinical outcomes of SARS-CoV-2 positive mothers and their newborns and comparison between subgroups based on newborn testing.

\begin{tabular}{|c|c|c|c|c|c|c|c|c|c|}
\hline \multirow{3}{*}{$\begin{array}{l}\text { Demographic \& clinical } \\
\text { characteristics }\end{array}$} & \multirow{2}{*}{\multicolumn{2}{|c|}{ Total M-positives }} & \multicolumn{6}{|c|}{ Mother/newborn dyads } & \multirow[t]{3}{*}{$p$ value $^{\mathrm{g}}$} \\
\hline & & & \multicolumn{2}{|c|}{ Positive/positive } & \multicolumn{2}{|c|}{ Positive/negative } & \multicolumn{2}{|c|}{ Positive/untested } & \\
\hline & $n$ & $\%$ & $n$ & $\%$ & $n$ & $\%$ & $n$ & $\%$ & \\
\hline Total \# deliveries & 289 & & 11 & $4 \%$ & 245 & $85 \%$ & 33 & $11 \%$ & \\
\hline \# Fetal demise & 3 & & 0 & $0 \%$ & 0 & $0 \%$ & 3 & $100 \%$ & \\
\hline Total \# mothers of living newborns & 286 & & 11 & $4 \%$ & 245 & $86 \%$ & 30 & $10 \%$ & \\
\hline \multicolumn{10}{|l|}{ Demographics } \\
\hline Age (median) & 29 & & 33 & & 29 & & 27 & & 0.1967 \\
\hline \multicolumn{10}{|l|}{ Gravida } \\
\hline Primigravida & 54 & $18 \%$ & 1 & $2 \%$ & 47 & $87 \%$ & 6 & $11 \%$ & 0.8851 \\
\hline Multigravida & 220 & $27 \%$ & 9 & $4 \%$ & 187 & $85 \%$ & 24 & $11 \%$ & \\
\hline \multicolumn{10}{|l|}{ Race/Ethnicity } \\
\hline Black / Non-Hispanic & 64 & $22 \%$ & 1 & $9 \%$ & 56 & $23 \%$ & 7 & $23 \%$ & $\mathrm{~h}$ \\
\hline Latino / Hispanic & 113 & $40 \%$ & 6 & $55 \%$ & 98 & $40 \%$ & 9 & $30 \%$ & \\
\hline Asian / Non-Hispanic & 31 & $11 \%$ & 0 & $0 \%$ & 25 & $10 \%$ & 6 & $20 \%$ & \\
\hline $\begin{array}{l}\text { Unspecified Race / Non- } \\
\text { Hispanic }\end{array}$ & 18 & $6 \%$ & 1 & $9 \%$ & 16 & $7 \%$ & 1 & $3 \%$ & \\
\hline $\begin{array}{l}\text { Unspecified Race / Other } \\
\text { Hispanic }\end{array}$ & 16 & $6 \%$ & 0 & $0 \%$ & 14 & $6 \%$ & 2 & $7 \%$ & \\
\hline White / Non-Hispanic & 10 & $4 \%$ & 1 & $9 \%$ & 9 & $4 \%$ & 0 & $0 \%$ & \\
\hline Unspecified Race \& Ethnicity & 7 & $2 \%$ & 0 & $0 \%$ & 7 & $3 \%$ & 0 & $0 \%$ & \\
\hline $\begin{array}{l}\text { Other Race \& Ethnicity } \\
\text { Combination }\end{array}$ & 27 & $9 \%$ & 2 & $18 \%$ & 20 & $8 \%$ & 5 & $17 \%$ & \\
\hline \multicolumn{10}{|l|}{ Socioeconomic factors } \\
\hline \multicolumn{10}{|l|}{ Insurance } \\
\hline Private & 36 & $13 \%$ & 2 & $18 \%$ & 32 & $13 \%$ & 2 & $7 \%$ & 0.6102 \\
\hline $\begin{array}{l}\text { Medicaid/ Medicaid } \\
\text { Managed Care }\end{array}$ & 248 & $87 \%$ & 9 & $82 \%$ & 211 & $86 \%$ & 28 & $93 \%$ & \\
\hline Other $^{\mathrm{b}}$ & 2 & $1 \%$ & 0 & $0 \%$ & 2 & $1 \%$ & 0 & $0 \%$ & \\
\hline \multicolumn{10}{|l|}{ NYC Borough } \\
\hline Bronx & 81 & $29 \%$ & 2 & $18 \%$ & 61 & $25 \%$ & 18 & $60 \%$ & $\mathrm{~h}$ \\
\hline Brooklyn & 82 & $29 \%$ & 2 & $18 \%$ & 74 & $31 \%$ & 6 & $20 \%$ & \\
\hline Manhattan & 9 & $3 \%$ & 0 & $0 \%$ & 7 & $3 \%$ & 2 & $7 \%$ & \\
\hline Queens & 110 & $39 \%$ & 7 & $64 \%$ & 99 & $41 \%$ & 4 & $13 \%$ & \\
\hline Staten Island & 0 & $0 \%$ & 0 & $0 \%$ & 0 & $0 \%$ & 0 & $0 \%$ & \\
\hline \multicolumn{10}{|l|}{ Health status } \\
\hline BMI (mean, median) & $31.9,30.9$ & & $28.4,28.1$ & & $32.1,31.4$ & & $31.5,29.5$ & & 0.1152 \\
\hline \multicolumn{10}{|l|}{ BMI, categorical } \\
\hline Underweight, $<18.5 \mathrm{~kg} / \mathrm{m} 2$ & 0 & $0 \%$ & 0 & $0 \%$ & 0 & $0 \%$ & 0 & $0 \%$ & 0.216 \\
\hline Normal, 18.5 to $<25 \mathrm{~kg} / \mathrm{m} 2$ & 20 & $8 \%$ & 2 & $20 \%$ & 15 & $7 \%$ & 3 & $10 \%$ & \\
\hline Overweight, 25 to $<30 \mathrm{~kg} / \mathrm{m} 2$ & 90 & $34 \%$ & 4 & $40 \%$ & 74 & $32 \%$ & 12 & $41 \%$ & \\
\hline Obese, $\geq 30 \mathrm{~kg} / \mathrm{m} 2$ & 157 & $59 \%$ & 4 & $40 \%$ & 139 & $61 \%$ & 14 & $48 \%$ & \\
\hline Asthma & 18 & $6 \%$ & 1 & $9 \%$ & 15 & $6 \%$ & 2 & $7 \%$ & 0.7326 \\
\hline Hypertension & 62 & $22 \%$ & 1 & $9 \%$ & 55 & $22 \%$ & 6 & $20 \%$ & 0.6843 \\
\hline Diabetes $^{\mathrm{a}}$ & 47 & $16 \%$ & 1 & $9 \%$ & 42 & $17 \%$ & 4 & $13 \%$ & 0.8184 \\
\hline No chronic disease & 180 & $63 \%$ & 8 & $73 \%$ & 152 & $62 \%$ & 20 & $67 \%$ & 0.9842 \\
\hline Only 1 of the three chronic diseases & 86 & $30 \%$ & 3 & $27 \%$ & 75 & $31 \%$ & 8 & $27 \%$ & \\
\hline 2 or more chronic diseases & 20 & $7 \%$ & 0 & $0 \%$ & 18 & $7 \%$ & 2 & $7 \%$ & \\
\hline
\end{tabular}


Table 1 (continued)

\begin{tabular}{|c|c|c|c|c|c|c|c|c|c|}
\hline \multirow{3}{*}{$\begin{array}{l}\text { Demographic \& clinical } \\
\text { characteristics }\end{array}$} & \multirow{2}{*}{\multicolumn{2}{|c|}{ Total M-positives }} & \multicolumn{6}{|c|}{ Mother/newborn dyads } & \multirow[t]{3}{*}{$p$ value } \\
\hline & & & \multicolumn{2}{|c|}{ Positive/positive } & \multicolumn{2}{|c|}{ Positive/negative } & \multicolumn{2}{|l|}{ Positive/untested } & \\
\hline & $n$ & $\%$ & $n$ & $\%$ & $n$ & $\%$ & $n$ & $\%$ & \\
\hline \multicolumn{10}{|l|}{ Maternal hospital course } \\
\hline Fever $(>=100.4 \mathrm{~F} / 38 \mathrm{C})$ & 69 & $24 \%$ & 1 & $9 \%$ & 65 & $27 \%$ & 3 & $10 \%$ & 0.0713 \\
\hline Chest Imaging & 53 & $19 \%$ & 1 & $9 \%$ & 51 & $21 \%$ & 1 & $3 \%$ & $0.0327^{\mathrm{i}}$ \\
\hline Maternal respiratory support & 83 & $29 \%$ & 2 & $18 \%$ & 73 & $30 \%$ & 8 & $27 \%$ & 0.7329 \\
\hline $\begin{array}{l}\text { Duration of ROM, hours, [mean, } \\
\text { median] }\end{array}$ & $6.4,2$ & & 3,3 & & $6.6,2$ & & $5.6,2$ & & 0.8041 \\
\hline \multicolumn{10}{|l|}{ Route of Delivery } \\
\hline C-section & 94 & $33 \%$ & 5 & $46 \%$ & 80 & $33 \%$ & 9 & $30 \%$ & 0.6352 \\
\hline Vaginal & 190.0 & $66 \%$ & 6 & $55 \%$ & 163 & $67 \%$ & 21 & $70 \%$ & \\
\hline Breastfeeding & 166 & $57 \%$ & 9 & $82 \%$ & 136 & $55 \%$ & 21 & $70 \%$ & 0.0711 \\
\hline Skin-to-skin & 103 & $36 \%$ & 4 & $36 \%$ & 88 & $35 \%$ & 11 & $37 \%$ & 1 \\
\hline $\begin{array}{l}\text { Mother hospital LOS, hours, [mean, } \\
\text { median] }\end{array}$ & $78.5,60$ & & 69,62 & & $81.4,61$ & & $58.7,55.5$ & & 0.1923 \\
\hline Total living newborns & 290 & & 11 & $4 \%$ & 249 & $86 \%$ & 30 & $10 \%$ & \\
\hline \multicolumn{10}{|l|}{ Newborn hospital course } \\
\hline $\begin{array}{l}\text { Gestational Age, week/day [mean, } \\
\text { median] }\end{array}$ & $384 / 7$ & $386 / 7$ & $373 / 7$ & $375 / 7$ & $382 / 7$ & $386 / 7$ & $382 / 7$ & $391 / 7$ & 0.2473 \\
\hline \multicolumn{10}{|l|}{ Gestational Age, Categorical ${ }^{\mathrm{c}}$} \\
\hline Extreme - Very Preterm & 8 & $3 \%$ & 1 & $9 \%$ & 6 & $2 \%$ & 1 & $3 \%$ & 0.1787 \\
\hline Preterm & 42 & $15 \%$ & 0 & $0 \%$ & 40 & $16 \%$ & 2 & $7 \%$ & \\
\hline Full Term & 240 & $83 \%$ & 10 & $91 \%$ & 203 & $82 \%$ & 27 & $90 \%$ & \\
\hline Birthweight, grams [mean, median] & $3060.5,3127.5$ & & $3026.5,3125$ & & $3061.6,3116$ & & $3063.6,3222.5$ & & 0.9010 \\
\hline \multicolumn{10}{|l|}{ Birthweight, Categorical $^{\mathrm{d}}$} \\
\hline VLBW & 6 & $2 \%$ & 1 & $9 \%$ & 4 & $2 \%$ & 1 & $3 \%$ & 0.1155 \\
\hline LBW & 26 & $9 \%$ & 0 & $0 \%$ & 24 & $10 \%$ & 2 & $7 \%$ & 0.801 \\
\hline 1 min Apgar [median] & 9 & & 9 & & 9 & & 9 & & 0.5901 \\
\hline 5 min apgar [median] & 9 & & 9 & & 9 & & 9 & & 0.3182 \\
\hline Newborn nursery & 168 & $58 \%$ & 8 & $73 \%$ & 148 & $59 \%$ & 12 & $40 \%$ & 0.0833 \\
\hline NICU admission ${ }^{\mathrm{e}}$ & 121 & $42 \%$ & 7 & $64 \%$ & 107 & $43 \%$ & 7 & $23 \%$ & $0.0389^{\mathrm{i}}$ \\
\hline NICU LOS, hours, [mean, median] & $222.1,65$ & & $218.7,50$ & & $229.3,67$ & & $116.4,83.0$ & & 0.3939 \\
\hline Newborn respiratory support & 46 & $16 \%$ & 2 & $18 \%$ & 39 & $16 \%$ & 5 & $17 \%$ & 0.8139 \\
\hline $\begin{array}{l}\text { Newborn hospital LOS hours } \\
\text { [mean, median] }\end{array}$ & $115.6,53$ & & $179.4,61$ & & $119.3,54$ & & $61.3,47$ & & $0.0040^{\mathrm{j}}$ \\
\hline $\begin{array}{l}\text { Never in NICU newborn } \text { LOS }^{\mathrm{f}} \\
\text { hours [mean, median] }\end{array}$ & $50.9,47$ & & $57.3,52$ & & $52.0,47$ & & $42.7,46$ & & 0.1557 \\
\hline
\end{tabular}

${ }^{a}$ Type 1 or Type $2 \mathrm{DM}$ or gestational diabetes.

${ }^{\mathrm{b}}$ Other includes medicare, medicare managed care, self-pay.

${ }^{c}$ Extreme preterm: <28 weeks; Very preterm: 28-32 weeks; Preterm: 33-37 weeks; Full Term: >37 weeks.

${ }^{\mathrm{d}}$ LBW: $<1500 \mathrm{~g}$; VLBW: $<2500 \mathrm{~g}$.

${ }^{\mathrm{e}}$ Newborn admitted to NICU at any time during birth encounter.

${ }^{\mathrm{f}}$ Newborn never admitted to NICU during birth encounter.

${ }^{\mathrm{g}}$ Fisher's exact for categorical variables, ANOVA for age, and Kruskal Wallis for other numerical variables.

${ }^{\text {h}}$ Fisher's unable to calculate.

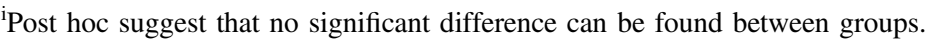

${ }^{j}$ Post hoc suggests that no significant difference can be found between P-N and P-P. However, LOS is significantly longer among P-P and P-N compared with P-U. 
Fig. 3 Practice variation during surge period.

A Breastfeeding rates B Skin-toskin rates $\mathbf{C}$ Maternal length of stay among SARS-CoV-2 positive mothers at $\mathrm{NYC} \mathrm{H}+\mathrm{H}$ facilities by week between March 1 and May 9, 2020 compared to baseline.

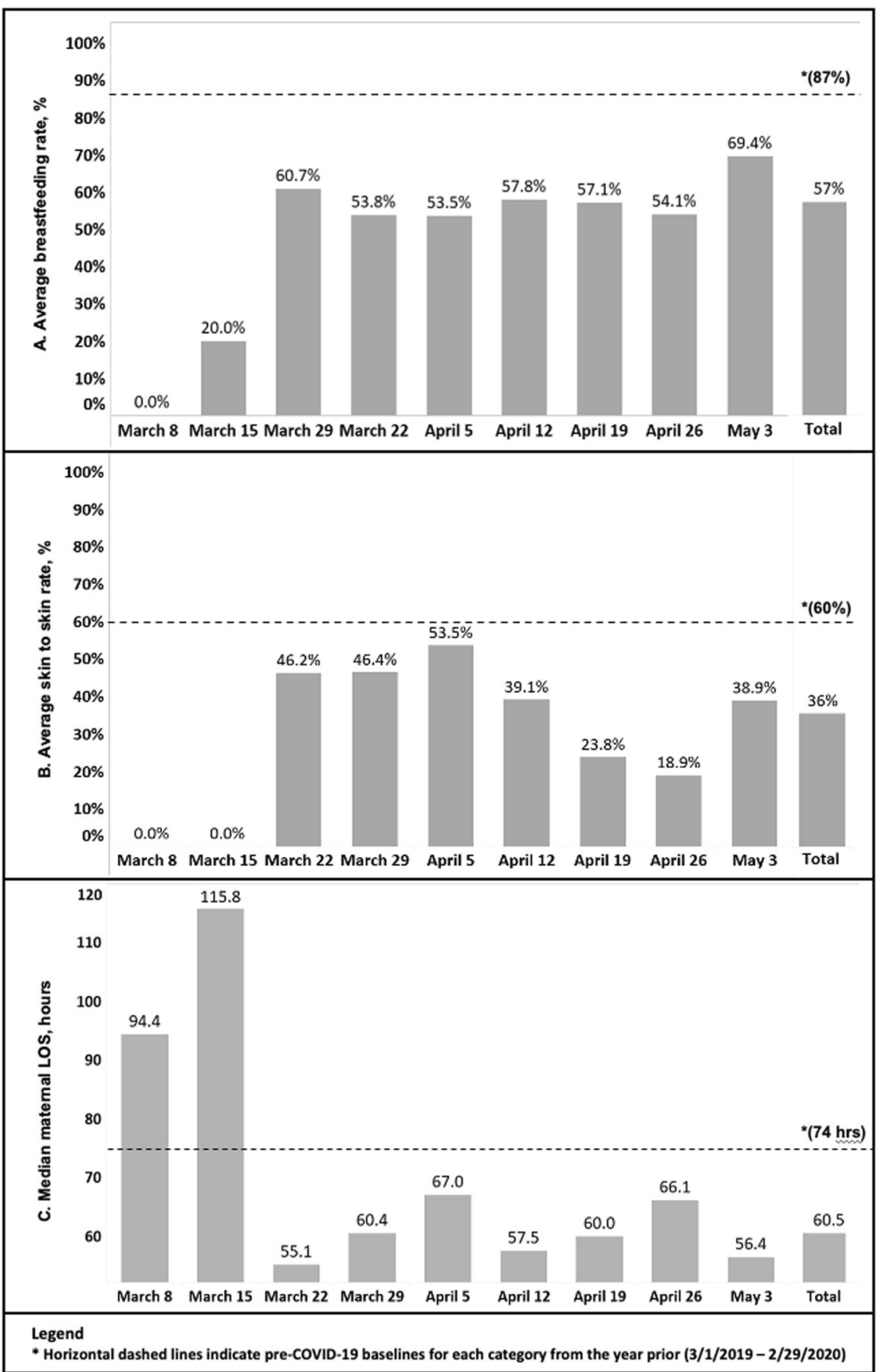

prioritized to get Cepheid tests as their results impacted hospital course and resources needed for management before, during, and after delivery.

AAP guidelines published on April 2, 2020 recommended testing newborns of M-positives at $\sim 24 \mathrm{~h}$ after birth and an optional second test, if clinically required, prior to discharge [4]. These guidelines did not address testing for newborns of M-untested or M-negatives. The clinical council for all 11 NICUs in our system communicated the varied approaches in real time as each facility adapted to the protocols, the shifting resources, and staffing models to protect providers and patients. In reality, over a third of all newborns tested were born to PUIs who later resulted as Mnegatives. Because of the unprecedented surge in general hospitalizations at some centers, there arose a need for not delaying discharge for the mother-baby dyad. $70 \%$ of all tested newborns were tested only once prior to discharge due to early newborn discharge, acute shortage of testing supplies or delay in obtaining mother's results. $41.2 \%$ (107/ 260) of newborns of M-positives were tested two or more times in basic accordance with these guidelines. $10 \%$ (30/290) of the newborns of M-positives were discharged 
Fig. 4 Testing timeline. SARS-CoV-2 testing timeline of 11 Positive/positive dyads.

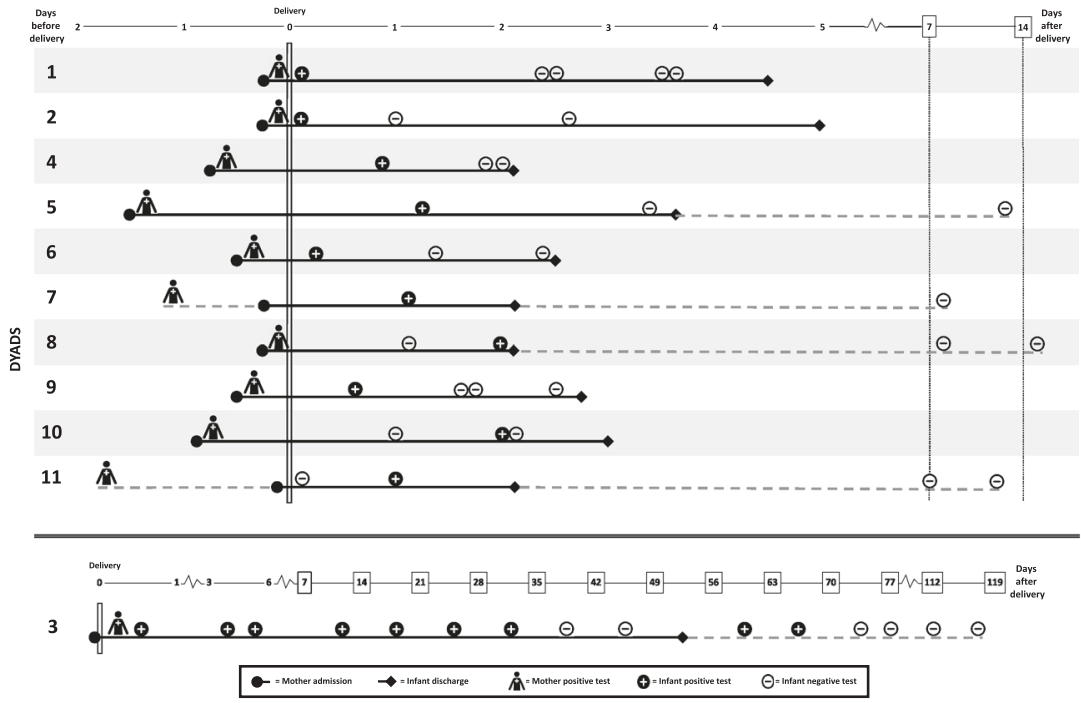

without being tested $(\mathrm{P} / \mathrm{U})$ due to either prolonged turnaround time of maternal test result or shortage of supplies.

The breastfeeding, skin-to-skin, and bathing practices also varied widely by week and between hospitals to adapt to COVID-19 protocols at each site and was affected by availability of maternal testing, timing of testing relative to delivery, and time until test result. Overall, the skin-to-skin rate was impacted not by the positivity but by the testing. The breastfeeding rate for $\mathrm{P} / \mathrm{P}$ dyads was higher and it was unclear if the positivity was impacted by breastfeeding, given that there was variation in approach to positive babies. Some facilities tested before and others after contact with the mother and breastfeeding. Other smaller NYC surge-period reports from hospitals that did not follow the contact restricting guidelines showed higher rates for these metrics. Of note, these studies reported no validated positive newborns [21, 22].

The length-of-stay decisions were dynamic and varied as expected. Early in the study, the length of stay was significantly longer since during this period only symptomatic mothers were being tested. With the uptake of universal testing, there was a clear trend toward shortened length of stay [5]. There was an effort to safely discharge the dyads as early as possible in the interest of reducing exposure and protecting the relatively healthy mothers and newborns. However, these efforts were countered by several barriers around safe discharge planning. These included follow up of results, completion of required newborn screenings, discharge to an asymptomatic caregiver, inability of our patients to get a ride home or inability to get in-person follow-up for bilirubin or weight checks.

The short-term outcomes while in the hospital of the three cohorts $(\mathrm{P} / \mathrm{P}, \mathrm{P} / \mathrm{N}$, and $\mathrm{P} / \mathrm{U})$ were favorable for the majority of cases. The overall length of stay for all tested $(\mathrm{P} / \mathrm{P}$ and $\mathrm{P} / \mathrm{N})$ newborns was prolonged due to the reasons stated above. The untested newborns were discharged at the expected average of 2.5 days. The $\mathrm{P} / \mathrm{P}$ newborns were more likely to be admitted to the NICU but the length of NICU stay for all the groups was similar. This is because the NICUs were often being used as the number of PUIs and resultant need for isolation grew. There was no significant difference in the need for newborn respiratory support between these groups, again reconfirming that NICU admission was not driven by need for clinical intervention.

Of note, P/P newborn \#3 was also the only newborn who tested positive for SARS-CoV-2 on more than one occasion from different samples. The remaining $\mathrm{P} / \mathrm{P}$ newborns all tested positive only once, and most had more than 2 followup negative tests. These findings can be explained either by transient viral colonization, contamination, or true viral infection with an abbreviated and mostly asymptomatic course.

There are limitations to this study. As universal testing for newborns of positive mothers was implemented in different ways at different hospitals, we cannot definitively assert a prevalence of positive newborns. We also cannot fully explain the variance in timing of infant positivity. There seems to be a trend in increase in positivity rate by the number of tests done. However, the newborns who tested positive were more likely to be re-tested and had a longer length of stay because of positivity. The lack of confirmatory anti-SARS-CoV-2 antibody testing is also a limiting factor as it could help explain the nature of infection causing positive rt-PCR testing results as well as insights into potential earlier or prolonged in utero exposure. In addition, the study is based on extraction of electronic health records and not manual chart review given the large number of patients. The study is over an abbreviated timeline of clinical assessment for these newborns. The uncomplicated course of these newborns, while promising, 
does not allow for evaluation of late presentation, long-term outcomes, or secondary outcomes such as delayed standard of care or unnecessary interventions. We plan to continue to monitor the follow-up of these newborns in order to assess the risk of possible emergence of COVID-19 related symptoms later in the newborn period as well as any unintended consequences of the COVID-19 diagnosis.

To our knowledge, this is the first study to investigate the impact of varied approaches to the management of the mother-baby dyads of M-positives. We also believe that our study describes the largest cohort of newborns with laboratory-confirmed SARS-CoV-2 infection.

Our study shows that varied testing, feeding, and isolation practices that adapted to national guidelines still resulted in favorable short-term outcomes for SARS-CoV-2 positive mothers and their newborns. We showed that there were no significant differences between dyads stratified by newborn testing. These findings are especially valuable as they show that high-risk populations can be safely and effectively treated in resource limited environments. As the pandemic continues to surge in new parts of the United States and resurge in previously affected areas, our findings will help inform healthcare systems towards effective management of dyads and allocation of resources.

Acknowledgements We thank Dr. Machelle Allen and the $\mathrm{NYCH}+\mathrm{H}$ COVID-19 Research Steering Committee for providing infrastructure, technical and administrative support. We thank Dr. Wendy Wilcox and the Women's Health Council for the clinical outcomes. We thank Hugo Teo and Carmen Farrow for their data extraction and reports. We thank Kenra Ford for guidance on clinical operations. We thank all members of the NYCH + H Perinatal COVID Research Subcommittee YM, IH, DW, Judith Chervenak MD, Daniel Lasser MD, Andrew Wiznia MD, Nichola Davis MD, Roopa Marcello MPH, and Joseph Masci MD for their assistance in project facilitation. We thank all of the following Neonatal Division Chiefs for providing insight into each facility's practice and adaptation during the COVID-19 surge: Dr. Ronald Bainbridge, Dr. John Casey, Dr. Alice Garner, Dr. Emad Ghaly, Dr. IH, Dr. Mary Marron-Corwin, Dr. Beth Nagourney, Dr. Chris Papazafiratou, Dr. UP, Dr. Ronald Saintonge, and Dr. Elena Wachtel.

Author contributions YM and CK were responsible for project ideation, data analysis, first draft of the paper and its revisions, data visualization, and paper preparation. TL performed data validation and statistical analyses, and along with KW contributed to data visualization and figure creation. AG assisted with writing the introduction. IH contributed to draft revision and tracking. HS, TS, and MR worked on data extraction and along with UP, AG, and DW contributed draft edits.

\section{Compliance with ethical standards}

Conflict of interest The authors declare no competing interests.

Publisher's note Springer Nature remains neutral with regard to jurisdictional claims in published maps and institutional affiliations.

\section{References}

1. Breslin N, Baptiste C, Gyamfi-Bannerman C, Miller R, Martinez $\mathrm{R}$, Bernstein $\mathrm{K}$, et al. COVID-19 infection among asymptomatic and symptomatic pregnant women: two weeks of confirmed presentations to an affiliated pair of New York City hospitals. Am J Obstet Gynecol MFM. 2020;2:100118 https://doi.org/10.1016/j. ajogmf.2020.100118. Epub 2020 Apr 9.

2. Muhidin S, Behboodi Moghadam Z, Vizheh M. Analysis of maternal coronavirus infections and neonates born to mothers with 2019-nCoV; a systematic review. Arch Acad Emerg Med. 2020;8: e49. Published 2020 Apr 15.

3. Juan J, Gil MM, Rong Z, Zhang Y, Yang H, Poon LC. Effects of coronavirus disease 2019 (COVID-19) on maternal, perinatal and neonatal outcomes: a systematic review. Ultrasound Obstet Gynecol. 2020;56:15-27. https://doi.org/10.1002/ uog. 22088.

4. Puopolo KM, Hudak ML, Kimberlin DW, Cummings J. Initial Guidance: management of infants born to mothers with COVID19. American Academy of Pediatrics Committee on fetus and newborn section on neonatal and perinatal medicine and committee on infectious disease. Initial guidance: management of infants born to mothers with COVID-19. 2020. https://www.tn. gov/content/dam/tn/health/documents/cedep/novel-coronavirus/ AAP_COVID-19-Initial-Newborn-Guidance.pdf.

5. Considerations for inpatient obstetric healthcare settings. Centers for disease control and prevention (2020), May 2020. https://www.cdc. gov/coronavirus/2019-ncov/hcp/inpatient-obstetric-healthcare-guida nce.html.

6. Evaluation and management considerations for neonates at risk for COVID-19. Centers for disease control and prevention (2020), May 2020. https://www.cdc.gov/coronavirus/2019-ncov/hcp/ca ring-for-newborns.html.

7. Novel coronavirus 2019 (COVID-19). American College of obstetrics and gynecology, June 2020. https://www.acog.org/ clinical/clinical-guidance/practice-advisory/articles/2020/03/ novel-coronavirus-2019.

8. Wilcox W, Bajaj K, Rossberg MC, Knight C, Wieland D, Malhotra Y. Lessons learnt in transitioning from universal screening to universal testing of pregnant patients for SARS-CoV-2 at the largest municipal health system in America. J Perinatol. 2021. https://doi.org/10.1038/s41372-020-00889-4.

9. Precit MR, Yee R, Anand V, Mongkolrattanothai K, Pandey U, Bard JD, et al. A case report of neonatal acute respiratory failure due to SARS-CoV-2. J Pediatr Infect Dis Soc. 2020;9:390-2. https://doi.org/10.1093/jpids/piaa064.

10. Dumpa V, Kamity R, Vinci AN, Noyola E, Noor A. Neonatal coronavirus 2019 (COVID-19) infection: a case report and review of literature. Cureus. 2020;12:e8165 https://doi.org/10.7759/ cureus.8165. Published 2020 May 17.

11. Di Mascio D, Khalil A, Saccone G, Rizzo F, Buca D, Liberati M, et al. Outcome of coronavirus spectrum infections (SARS, MERS, COVID-19) during pregnancy: a systematic review and metaanalysis. Am J Obstet Gynecol MFM. 2020;2:100107 https://doi. org/10.1016/j.ajogmf.2020.100107.

12. World Health Organization. Pregnancy and pandemic influenza A (H1N1) 2009: information for programme managers and clinicians. July 2010; https://www.who.int/csr/resources/publications/ swineflu/h1n1_guidance_pregnancy.pdf?ua $=1$.

13. Cobas $^{\circledast}$ SARS-CoV-2. Qualitative assay for use on the Cobas ${ }^{\varpi}$ 6800/8800 Systems. Food and drug administration, May 2020; https://www.fda.gov/media/136049/download.

14. Xpert ${ }^{\circledast}$ Xpress SARS-CoV-2. Instructions for use. Food and drug administration. https://www.fda.gov/media/136314/download. 
15. Emergency use authorization (EUA) summary. Covid-19 RT_PCR test. Food and drug administration, November 2020; https://www. fda.gov/media/136151/download.

16. Chandra S, Tripathi AK, Mishra S, Amzarul M, Vaish AK. Physiological changes in hematological parameters during pregnancy. Indian J Hematol Blood Transfus. 2012;28:144-6. https://doi.org/10.1007/s12288-012-0175-6.

17. Wu Z, McGoogan JM. Characteristics of and important lessons from the Coronavirus disease 2019 (COVID-19) outbreak in China: summary of a report of 72314 cases from the Chinese Center for Disease Control and Prevention. JAMA. 2020;323:1239-42. https://doi.org/10.1001/jama.2020.2648.

18. Gupta A, Malhotra Y, Patil U, Muradas AR, Lee WT, Krammer F, et al. In utero vertical transmission of Coronavirus Disease 2019 in a severely ill 29-week preterm infant. AJP Rep. 2020;10: e270-e274. https://doi.org/10.1055/s-0040-1715177.

19. Maternal mortality and disparate racial outcomes. Health.ny.gov. http://www.health.ny.gov/community/adults/women/task_force_ maternal_mortality/docs/maternal_mortality_report.pdf. Published March 2019.

20. Patil UP, Maru S, Krishnan P, Carroll-Bennett R, Sanchez J, Noble L, et al. Newborns of COVID-19 mothers: short-term outcomes of colocating and breastfeeding from the pandemic's epicenter. J Perinatol. 2020;40:1455-8. https://doi.org/10.1038/ s41372-020-0765-3.

21. Salvatore CM, Han JY, Acker KP, Tiwari P, Jin J, Brandler M, et al. Neonatal management and outcomes during the COVID-19 pandemic: an observation cohort study. Lancet Child Adolesc Health. 2020;4:721-7. https://doi.org/10.1016/S2352-4642(20) 30235-2. Epub 2020 Jul 23.

22. Dumitriu D, Emeruwa UN, Hanft E, Liao GV, Ludwig E, Walzer L, et al. Outcomes of neonates born to mothers with Severe Acute Respiratory Syndrome Coronavirus 2 infection at a large medical center in New York City. JAMA Pediatr. (2020 Oct). https://doi.org/10.1001/jamapedia trics.2020.4298. 